H. M. Srivastava and S. Owa

Nagoya Math. J.

Vol. 106 (1987), 1-28

\title{
SOME CHARACTERIZATION AND DISTORTION THEOREMS INVOLVING FRACTIONAL CALCULUS, GENERALIZED HYPERGEOMETRIC FUNCTIONS, HADAMARD PRODUCTS, LINEAR OPERATORS, AND CERTAIN SUBCLASSES OF ANALYTIC FUNCTIONS*
}

\author{
H. M. SRIVASTAVA AND SHIGEYOSHI OWA
}

\begin{abstract}
By using a certain linear operator defined by a Hadamard product or convolution, several interesting subclasses of analytic functions in the unit disk are introduced and studied systematically. The various results presented here include, for example, a number of coefficient estimates and distortion theorems for functions belonging to these subclasses, some interesting relationships between these subclasses, and a wide variety of characterization theorems involving a certain functional, some general functions of hypergeometric type, and operators of fractional calculus. Some of the coefficient estimates obtained here are fruitfully applied in the investigation of certain subclasses of analytic functions with fixed finitely many coefficients.
\end{abstract}

\section{§1. Introduction and Definitions}

Let $\mathscr{A}$ denote the class of functions of the form

$$
f(z)=\sum_{n=0}^{\infty} a_{n+1} z^{n+1} \quad\left(a_{1}=1\right)
$$

which are analytic in the unit disk $\mathscr{U}=\{z:|z|<1\}$. Then a function $f(z)$ belonging to $\mathscr{A}$ is said to be in the class $\mathscr{N}(\alpha)$ if it satisfies the inequality

$$
\operatorname{Re}\left\{\frac{z f^{\prime}(z)}{f(z)}\right\}>-\alpha \quad(z \in \mathscr{U})
$$

Received June 12, 1985.

Revised February 19, 1986.

* The present investigation was carried out at the University of Victoria while the second author was on study leave from Kinki University, Osaka, Japan.

This work was supported, in part, by the Natural Sciences and Engineering Research Council of Canada under Grant A-7353. 
for $\alpha>-1$. On the other hand, a function $f(z)$ belonging to $\mathscr{A}$ is said to be in the class $\mathscr{W}(\alpha)$ if it satisfies the inequality

$$
\operatorname{Re}\left\{1+\frac{z f^{\prime \prime}(z)}{f^{\prime}(z)}\right\}>-\alpha \quad(z \in \mathscr{U})
$$

for $\alpha>-1$. It is easily observed that

$$
f(z) \in \mathscr{W}(\alpha) \Longleftrightarrow z f^{\prime}(z) \in \mathscr{V}(\alpha) ; \mathscr{W}(\alpha) \subset \mathscr{V}(\alpha) .
$$

Throughout this paper, it should be understood that functions such as $z f^{\prime}(z) / f(z)$ and $z f^{\prime \prime}(z) / f^{\prime}(z)$, which have removable singularities at $z=0$, have had these singularities removed in statements like (1.2) and (1.3).

Let $\alpha_{j}(j=1, \cdots, p)$ and $\beta_{j}(j=1, \cdots, q)$ be complex numbers with

$$
\beta_{j} \neq 0,-1,-2, \cdots ; j=1, \cdots, q .
$$

Then the generalized hypergeometric function ${ }_{p} F_{q}(z)$ is defined by (cf. $[16$, p. 33]).

$$
\begin{aligned}
{ }_{p} F_{q}(z) & \equiv{ }_{p} F_{q}\left(\alpha_{1}, \cdots, \alpha_{p} ; \beta_{1}, \cdots, \beta_{q} ; z\right) \\
& =\sum_{n=0}^{\infty} \frac{\left(\alpha_{1}\right)_{n} \cdots\left(\alpha_{p}\right)_{n}}{\left(\beta_{1}\right)_{n} \cdots\left(\beta_{q}\right)_{n}} \frac{z^{n}}{n !} \quad(p \leqq q+1),
\end{aligned}
$$

where $(\lambda)_{n}$ is the Pochhammer symbol defined by

$$
(\lambda)_{n}=\left\{\begin{array}{cl}
1, & \text { if } n=0 \\
\lambda(\lambda+1) \cdots(\lambda+n-1), & \text { if } n \in \mathscr{N}=\{1,2,3, \cdots\} .
\end{array}\right.
$$

We note that the ${ }_{p} F_{q}$ series in (1.5) converges absolutely for $|\boldsymbol{z}|<\infty$ if $p<q+1$, and for $z \in \mathscr{U}$ if $p=q+1$. Furthermore, if we set

$$
\omega=\sum_{j=1}^{q} \beta_{j}-\sum_{j=1}^{p} \alpha_{j},
$$

it is known (see, e.g., [16, p. 34]) that the ${ }_{p} F_{q}$ series, with $p=q+1$, is absolutely convergent for $|z|=1$ if $\operatorname{Re}(\omega)>0$, and conditionally convergent for $|z|=1(z \neq 1)$ if

$$
-1<\operatorname{Re}(\omega) \leqq 0
$$

For the functions $f_{i}(z)(i=1,2)$ defined by

$$
f_{i}(z)=\sum_{n=0}^{\infty} a_{i, n+1} z^{n+1} \quad\left(a_{i, 1}=1 ; i=1,2\right),
$$

let $f_{1} * f_{2}(z)$ denote the Hadamard product or convolution of $f_{1}(z)$ and $f_{2}(z)$, defined by 


$$
f_{1} * f_{2}(z)=\sum_{n=0}^{\infty} a_{1, n+1} a_{2, n+1} z^{n+1} .
$$

Now define the function $\phi(a, c)$ by

$$
\phi(a, c ; z)=\sum_{n=0}^{\infty} \frac{(a)_{n}}{(c)_{n}} z^{n+1} \quad(c \neq 0,-1,-2, \cdots ; z \in \mathscr{U}),
$$

so that $\phi(a, c)$ is an incomplete Beta function with [cf. Equation (1.5)]

$$
\phi(a, c ; z)=z_{2} F_{1}(1, a ; c ; z) .
$$

Corresponding to the function $\phi(a, c)$, Carlson and Shaffer [2] defined a linear operator $\mathscr{L}(a, c)$ on $\mathscr{A}$ by the convolution [2, p. 738, Equation (2.2)]

$$
\mathscr{L}(a, c) f=\phi(a, c) * f
$$

for $f(z) \in \mathscr{A}$. Clearly, $\mathscr{L}(a, c)$ maps $\mathscr{A}$ onto itself, and $\mathscr{L}(c, a)$ is an inverse of $\mathscr{L}(a, c)$, provided that

$$
a \neq 0,-1,-2, \cdots .
$$

Furthermore, $\mathscr{L}(a, a)$ is the identity operator, and

$$
\mathscr{W}(\alpha)=\mathscr{L}(1,2) \mathscr{V}(\alpha) \quad(\alpha>-1)
$$

or, inversely,

$$
\mathscr{V}(\alpha)=\mathscr{L}(2,1) \mathscr{W}(\alpha) \quad(\alpha>-1) .
$$

A function $f(z)$ belonging to $\mathscr{A}$ is said to be in the class $\mathscr{V}(\alpha, c ; \alpha)$ if $\mathscr{L}(a, c) f$ is an element of $\mathscr{V}(\alpha)$ for $\alpha>-1$. Further, a function $f(z)$ belonging to $\mathscr{A}$ is said to be in the class $\mathscr{W}(a, c ; \alpha)$ if $z f^{\prime}(z)$ is an element of $\mathscr{V}(a, c ; \alpha)$ for $\alpha>-1$. Then it is easily verified that [cf. Equations (1.13) and (1.14)]

$$
\begin{gathered}
\mathscr{W}(a, c ; \alpha)=\mathscr{L}(1,2) \mathscr{V}(a, c ; \alpha), \\
\mathscr{V}(a, c ; \alpha)=\mathscr{L}(2,1) \mathscr{W}(a, c ; \alpha), \\
\mathscr{W}(\alpha)=\mathscr{W}(a, a ; \alpha)=\mathscr{L}(1,2) \mathscr{V}(a, a ; \alpha),
\end{gathered}
$$

and

$$
\mathscr{V}(\alpha)=\mathscr{V}(a, a ; \alpha)=\mathscr{L}(2,1) \mathscr{W}(a, a ; \alpha) .
$$

A single-valued function $f(z)$ is said to be univalent in a domain $\mathscr{D}$ if it never takes on the same value twice, that is, if $f\left(z_{1}\right)=f\left(z_{2}\right)$ for $z_{1}$, $z_{2} \in \mathscr{D}$ implies that $z_{1}=z_{2}$. A set $\mathscr{E}$ is said to be starlike with respect to 
$w_{0} \in \mathscr{E}$ if the line segment joining $w_{0}$ to every other point $w \in \mathscr{E}$ lies entirely in $\mathscr{E}$. If a function $f(z)$ maps $\mathscr{D}$ onto a domain that is starlike with respect to $w_{0}$, then $f(z)$ is said to be starlike with respect to $w_{0}$. In particular, if $w_{0}$ is the origin, then we say that $f(z)$ is a starlike function in $\mathscr{D}$. Further, a set $\mathscr{E}$ is said to be convex if the line segment joining any two points of $\mathscr{E}$ lies entirely in $\mathscr{E}$. If a function $f(z)$ maps $\mathscr{D}$ onto a convex domain, then we say that $f(z)$ is a convex function in $\mathscr{D}$.

Now let $\mathscr{S}$ denote the class of all functions in $\mathscr{A}$ which are univalent in the unit disk $\mathscr{U}$. Then a function $f(z)$ belonging to $\mathscr{S}$ is said to be starlike of order $\alpha$ if and only if

$$
\operatorname{Re}\left\{\frac{z f^{\prime}(z)}{f(z)}\right\}>\alpha \quad(z \in \mathscr{U})
$$

for some $\alpha(0 \leqq \alpha<1)$. We denote by $\mathscr{S}^{*}(\alpha)$ the class of all functions in $\mathscr{S}$ which are starlike of order $\alpha$.

A function $f(z)$ belonging to $\mathscr{S}$ is said to be convex of order $\alpha$ if and only if

$$
\operatorname{Re}\left\{1+\frac{z f^{\prime \prime}(z)}{f^{\prime}(z)}\right\}>\alpha \quad(z \in \mathscr{U})
$$

for some $\alpha(0 \leqq \alpha<1)$. We denote by $\mathscr{K}(\alpha)$ the class of all functions in $\mathscr{S}$ which are convex of order $\alpha$.

We note that $f(z) \in \mathscr{K}(\alpha)$ if and only if $z f^{\prime}(z) \in \mathscr{S}^{*}(\alpha)$. We also have

$$
\begin{gathered}
\mathscr{S}^{*}(\alpha) \subseteq \mathscr{S}^{*}(0) \equiv \mathscr{S}^{*}, \\
\mathscr{K}(\alpha) \subseteq \mathscr{K}(0) \equiv \mathscr{K},
\end{gathered}
$$

and

$$
\mathscr{K}(\alpha) \subset \mathscr{S}^{*}(\alpha) \subset \mathscr{S}
$$

for $0 \leqq \alpha<1$.

The classes $\mathscr{S}^{*}(\alpha)$ and $\mathscr{K}(\alpha)$ were first introduced by Robertson [11], and were studied subsequently by Schild [13], MacGregor [7], Pinchuk [10], Jack [4], and others (cf., e.g., [9]). Indeed, from the above definitions of the various subclasses of $\mathscr{A}$, it follows readily that

$$
\mathscr{S}^{*}(\alpha) \subset \mathscr{V}(\beta) \subset \mathscr{A}
$$

and

$$
\mathscr{K}(\alpha) \subset \mathscr{W}(\beta) \subset \mathscr{A}
$$


for $0 \leqq \alpha<1$ and $-1<-\alpha \leqq \beta$.

Finally, let $\mathscr{T}$ be the subclass of $\mathscr{A}$ consisting of functions of the form

$$
f(z)=\sum_{n=0}^{\infty} a_{n+1} z^{n+1} \quad\left(a_{1}=1 ; a_{n+1} \leqq 0, \forall n \in \mathscr{N}\right),
$$

where, as also in (1.6), $\mathscr{N}=\{1,2,3, \cdots\}$, and denote by $\mathscr{V}_{0}(\alpha)$ and $\mathscr{W}_{0}(\alpha)$ the classes obtained, respectively, by taking the intersections of $\mathscr{V}(\alpha)$ and $\mathscr{W}(\alpha)$ with $\mathscr{T}$; that is,

$$
\mathscr{V}_{0}(\alpha)=\mathscr{V}(\alpha) \cap \mathscr{T} \quad(\alpha>-1)
$$

and

$$
\mathscr{W}_{0}(\alpha)=\mathscr{W}(\alpha) \cap \mathscr{T} \quad(\alpha>-1)
$$

Also let

$$
\mathscr{V}_{0}(a, c ; \alpha)=\mathscr{V}(a, c ; \alpha) \cap \mathscr{T} \quad(\alpha>-1)
$$

and

$$
\mathscr{W}_{0}(a, c ; \alpha)=\mathscr{W}(a, c ; \alpha) \cap \mathscr{T} \quad(\alpha>-1) .
$$

Silverman [14] studied the subclasses $\mathscr{T}^{*}(\alpha)$ and $\mathscr{C}(\alpha)$ of $\mathscr{S}$ defined by

$$
\mathscr{T}^{*}(\alpha)=\mathscr{S}^{*}(\alpha) \cap \mathscr{T} \quad(0 \leqq \alpha<1)
$$

and

$$
\mathscr{C}(\alpha)=\mathscr{K}(\alpha) \cap \mathscr{T} \quad(0 \leqq \alpha<1),
$$

so that, upon comparison with the definitions (1.27) to (1.30), we have

$$
\mathscr{T}^{*}(\alpha) \subset \mathscr{V}_{0}(a, a ; \beta)=\mathscr{V}_{0}(\beta) \subset \mathscr{A}
$$

and

$$
\mathscr{C}(\alpha) \subset \mathscr{W}_{0}(a, a ; \beta)=\mathscr{W}_{0}(\beta) \subset \mathscr{A}
$$

for $0 \leqq \alpha<1$ and $-1<-\alpha \leqq \beta$.

In the present paper, we prove a number of coefficient estimates and distortion theorems for functions belonging to the various subclasses of $\mathscr{A}$ defined above, and derive some interesting relationships involving these subclasses. For these subclasses we also give a wide variety of characterization theorems and numerous other applications involving a certain functional, some general functions of hypergeometric type, and operators 
of fractional calculus. By using some of the coefficient estimates obtained in Section 2, certain further subclasses of analytic functions with fixed finitely many coefficients are studied systematically.

\section{§2. Coefficient estimates}

The various coefficient estimates (given by Theorem 2.1 to Theorem 2.4 below) lay the foundation of our systematic study of the subclasses of $\mathscr{A}$ defined in the preceding section.

THEOREM 2.1. Let the function $f(z)$ be defined by (1.1). If

$$
\sum_{n=1}^{\infty} \frac{(2+\alpha)_{n}}{(1+\alpha)_{n}}\left|\frac{(a)_{n}}{(c)_{n}}\right|\left|a_{n+1}\right| \leqq 1
$$

for $\alpha>-1$. then $f(z) \in \mathscr{V}(a, c ; \alpha)$. The result (2.1) is sharp.

Proof. We need to prove that (2.1) implies

$$
\operatorname{Re}\left\{\frac{z[\mathscr{L}(a, c) f(z)]^{\prime}}{\mathscr{L}(a, c) f(z)}\right\}>-\alpha \quad(z \in \mathscr{U})
$$

for $\alpha>-1$. And, in order to establish the inequality (2.2), it is sufficient to deduce from (2.1) that

$$
\left|\frac{z[\mathscr{L}(a, c) f(z)]^{\prime}}{\mathscr{L}(a, c) f(z)}-1\right|<1+\alpha \quad(z \in \mathscr{U})
$$

for $\alpha>-1$.

Indeed, since

$$
\mathscr{L}(a, c) f(z)=\sum_{n=0}^{\infty} \frac{(a)_{n}}{(c)_{n}} a_{n+1} z^{n+1} \quad\left(a_{1}=1\right),
$$

we have

$$
\begin{aligned}
& \left|\frac{z[\mathscr{L}(a, c) f(z)]^{\prime}}{\mathscr{L}(a, c) f(z)}-1\right| \\
& \quad \leqq \frac{\sum_{n=1}^{\infty} n\left|\frac{(a)_{n}}{(c)_{n}}\right|\left|a_{n+1}\right|}{1-\sum_{n=1}^{\infty}\left|\frac{(a)_{n}}{(c)_{n}}\right|\left|a_{n+1}\right|} \leqq 1+\alpha,
\end{aligned}
$$

provided that

$$
\sum_{n=1}^{\infty} n\left|\frac{(a)_{n}}{(c)_{n}}\right|\left|a_{n+1}\right| \leqq(1+\alpha)\left(1-\sum_{n=1}^{\infty}\left|\frac{(a)_{n}}{(c)_{n}}\right|\left|a_{n+1}\right|\right),
$$


which is equivalent to (2.1).

Clearly, the result (2.1) is sharp for the functions given by

$$
f(z)=z+\frac{(1+\alpha)_{n}(c)_{n}}{(2+\alpha)_{n}(a)_{n}} z^{n+1} \quad(n \in \mathscr{N}),
$$

which completes the proof of Theorem 2.1.

Corollary 2.1. Let the function $f(z)$ be defined by (1.1). If

$$
\sum_{n=1}^{\infty} \frac{(2+\alpha)_{n}}{(1+\alpha)_{n}}\left|a_{n+1}\right| \leqq 1
$$

for $\alpha>-1$, then $f(z) \in \mathscr{V}(\alpha)$. The result (2.8) is sharp for the functions given by

$$
f(z)=z+\frac{(1+\alpha)_{n}}{(2+\alpha)_{n}} z^{n+1} \quad(n \in \mathscr{N}) .
$$

Remark 2.1. In its special case when $-1<\alpha \leqq 0$, Corollary 2.1 yields the corresponding result for the class $\mathscr{S}^{*}(\alpha)$ due to Silverman [14, p. 110, Theorem 1].

THEOREM 2.2. Let the function $f(z)$ be defined by (1.1). If

$$
\sum_{n=1}^{\infty} \frac{(2)_{n}(2+\alpha)_{n}}{(1)_{n}(1+\alpha)_{n}}\left|\frac{(a)_{n}}{(c)_{n}}\right|\left|a_{n+1}\right| \leqq 1
$$

for $\alpha>-1$, then $f(z) \in \mathscr{W}(a, c ; \alpha)$. The result (2.10) is sharp.

Proof. Since $f(z) \in \mathscr{W}(a, c ; \alpha)$ if and only if

$$
z f^{\prime}(z)=\mathscr{L}(2,1) f(z) \in \mathscr{V}(a, c ; \alpha),
$$

we have the theorem if we replace $a_{n+1}$ in Theorem 2.1 by $\left[(2)_{n} a_{n+1}\right] /(1)_{n}$. Further, the result (2.10) is sharp for the functions given by

$$
f(z)=z+\frac{(1)_{n}(1+\alpha)_{n}(c)_{n}}{(2)_{n}(2+\alpha)_{n}(a)_{n}} z^{n+1} \quad(n \in \mathscr{N}) .
$$

Corollary 2.2. Let the function $f(z)$ be defined by (1.1). If

$$
\sum_{n=1}^{\infty} \frac{(2)_{n}(2+\alpha)_{n}}{(1)_{n}(1+\alpha)_{n}}\left|a_{n+1}\right| \leqq 1
$$

for $\alpha>-1$, then $f(z) \in \mathscr{W}(\alpha)$. The result (2.12) is sharp for the functions given by 


$$
f(z)=z+\frac{(1)_{n}(1+\alpha)_{n}}{(2)_{n}(2+\alpha)_{n}} z^{n+1} \quad(n \in \mathscr{N}) .
$$

Remark 2.2. If, in Corollary 2.2, we assume that $-1<\alpha \leqq 0$, we shall obtain the corresponding result for the class $\mathscr{K}(\alpha)$ due to Silverman [14, p. 110, Corollary].

For the classes $\mathscr{V}_{0}(a, c ; \alpha)$ and $\mathscr{W}_{0}(a, c ; \alpha)$, we next prove Theorem 2.3 and Theorem 2.4 below.

THEOREM 2.3. Let the function $f(z)$ be defined by (1.26). Then $f(z) \in$ $\mathscr{V}_{0}(a, c ; \alpha)$ if and only if

$$
\sum_{n=1}^{\infty} \frac{(2+\alpha)_{n}(a)_{n}}{(1+\alpha)_{n}(c)_{n}}\left|a_{n+1}\right| \leqq 1
$$

for $\alpha>-1$, where $(a)_{n} /(c)_{n}>0$. The result (2.14) is sharp.

Proof. Assume that (2.14) holds true. Then it follows immediately from Theorem 2.1 that $f(z) \in \mathscr{V}_{0}(a, c ; \alpha)$.

Conversely, assume that $f(z) \in \mathscr{V}_{0}(a, c ; \alpha)$. Then, by definition,

$$
\begin{aligned}
\operatorname{Re} & \left\{\frac{z[\mathscr{L}(a, c) f(z)]^{\prime}}{\mathscr{L}(a, c) f(z)}\right\} \\
& =\operatorname{Re}\left\{\frac{\sum_{n=0}^{\infty} \frac{(2)_{n}(a)_{n}}{(1)_{n}(c)_{n}} a_{n+1} z^{n+1}}{\sum_{n=0}^{\infty} \frac{(a)_{n}}{(c)_{n}} a_{n+1} z^{n+1}}\right\}>-\alpha
\end{aligned}
$$

for $\alpha>-1$ and $z \in \mathscr{U}$. Choose values of $z$ on the real axis so that

$$
\frac{z[\mathscr{L}(a, c) f(z)]^{\prime}}{\mathscr{L}(a, c) f(z)}
$$

is real. Upon clearing the denominator in (2.15) and letting $z \rightarrow 1^{-}$, we get

$$
\begin{aligned}
1- & \sum_{n=1}^{\infty} \frac{(2)_{n}(a)_{n}}{(1)_{n}(c)_{n}}-a_{n+1} \mid \\
& \geqq \alpha\left(\sum_{n=1}^{\infty} \frac{(a)_{n}}{(c)_{n}}\left|a_{n+1}\right|-1\right),
\end{aligned}
$$

which implies (2.14). Further, by taking the functions defined by

$$
f(z)=z-\frac{(1+\alpha)_{n}(c)_{n}}{(2+\alpha)_{n}(a)_{n}} z^{n+1} \quad(n \in \mathscr{N})
$$


we can prove that the result (2.14) is sharp.

Corollary 2.3. Let the function $f(z)$ defined by (1.26) be in the class $\mathscr{V}_{0}(a, c ; \alpha)$ with $(a)_{n} /(c)_{n}>0$. Then

$$
\left|a_{n+1}\right| \leqq \frac{(1+\alpha)_{n}(c)_{n}}{(2+\alpha)_{n}(a)_{n}} \quad(n \in \mathscr{N})
$$

The result (2.18) is sharp for the functions $f(z)$ given by (2.17).

Corollary 2.4. Let the function $f(z)$ be defined by (1.26). Then $f(z)$ $\in \mathscr{V}_{0}(\alpha)$ if and only if

$$
\sum_{n=1}^{\infty} \frac{(2+\alpha)_{n}}{(1+\alpha)_{n}}\left|a_{n+1}\right| \leqq 1
$$

for $\alpha>-1$. The result (2.19) is sharp for the functions given by

$$
f(z)=z-\frac{(1+\alpha)_{n}}{(2+\alpha)_{n}} z^{n+1} \quad(n \in \mathscr{N}) .
$$

Remark 2.3. For $\alpha$ constrained by $-1<\alpha \leqq 0$, Corollary 2.4 corresponds to a result for the class $\mathscr{T}^{*}(\alpha)$ proved by Silverman [14, p. 110, Theorem 2].

THEOREM 2.4. Let the function $f(z)$ be defined by (1.26). Then $f(z) \in$ $\mathscr{W}_{0}(a, c ; \alpha)$ if and only if

$$
\sum_{n=1}^{\infty} \frac{(2)_{n}(2+\alpha)_{n}(\alpha)_{n}}{(1)_{n}(1+\alpha)_{n}(c)_{n}}\left|a_{n+1}\right| \leqq 1
$$

for $\alpha>-1$, where $(a)_{n} /(c)_{n}>0$. The result (2.21) is sharp.

Proof. The proof of Theorem 2.4 is much akin to that of Theorem 2.3, and we skip the details involved. Equality in (2.21) is indeed attained by the functions given by

$$
f(z)=z-\frac{(1)_{n}(1+\alpha)_{n}(c)_{n}}{(2)_{n}(2+\alpha)_{n}(a)_{n}} z^{n+1} \quad(n \in \mathscr{N}) .
$$

Corollary 2.5. Let the function $f(z)$ defined by (1.26) be in the class $\mathscr{W}_{0}(a, c ; \alpha)$ with $(a)_{n} /(c)_{n}>0$. Then

$$
\left|a_{n+1}\right| \leqq \frac{(1)_{n}(1+\alpha)_{n}(c)_{n}}{(2)_{n}(2+\alpha)_{n}(\alpha)_{n}} \quad(n \in \mathscr{N}) .
$$

The result (2.23) is sharp for the fun tions $f(z)$ given by (2.22). 
Corollary 2.6. Let the function $f(z)$ be defined by (1.26). Then $f(z) \in$ $\mathscr{W}_{0}(\alpha)$ if and only if

$$
\sum_{n=1}^{\infty} \frac{(2)_{n}(2+\alpha)_{n}}{(1)_{n}(1+\alpha)_{n}}\left|a_{n+1}\right| \leqq 1
$$

for $\alpha>-1$. The result (2.24) is sharp for the functions given by

$$
f(z)=z-\frac{(1)_{n}(1+\alpha)_{n}}{(2)_{n}(2+\alpha)_{n}} z^{n+1} \quad(n \in \mathscr{N}) .
$$

Remark 2.4. Corollary 2.6 corresponds, for $-1<\alpha \leqq 0$, to a result for the class $\mathscr{C}(\alpha)$ given by Silverman [14, p. 111, Corollary 2].

\section{§3. Distortion theorems}

With a view to determining the extreme points of the classes $\mathscr{V}_{0}(a, c ; \alpha)$ and $\mathscr{W}_{0}(a, c ; \alpha)$, we first prove

Lemma 3.1. The class $\mathscr{V}_{0}(a, c ; \alpha)$ with $(a)_{n} /(c)_{n}>0$ is convex considered as a linear space over the field of real numbers.

Proof. Let the functions $f_{i}(z)(i=1,2)$ defined by [cf. Equation (1.8)]

$$
f_{i}(z)=\sum_{n=0}^{\infty} a_{i, n+1} z^{n+1} \quad\left(a_{i, 1}=1 ; a_{i, n+1} \leqq 0, \forall n \in \mathscr{N}\right)
$$

be in the class $\mathscr{V}_{0}(a, c ; \alpha)$. Then we need only prove that the function

$$
\lambda f_{1}(z)+(1-\lambda) f_{2}(z) \quad(0 \leqq \lambda \leqq 1)
$$

is also in the class $\mathscr{V}_{0}(a, c ; \alpha)$. Note that

$$
\begin{aligned}
\lambda f_{1}(z) & +(1-\lambda) f_{2}(z) \\
= & z+\sum_{n=1}^{\infty}\left(\lambda a_{1, n+1}+(1-\lambda) a_{2, n+1}\right) z^{n+1},
\end{aligned}
$$

which, by virtue of Theorem 2.3 , yields

$$
\begin{aligned}
& \sum_{n=1}^{\infty} \frac{(2+\alpha)_{n}(a)_{n}}{(1+\alpha)_{n}(c)_{n}}\left(\lambda\left|a_{1, n+1}\right|+(1-\lambda)\left|a_{2, n+1}\right|\right) \\
& \quad=\lambda \sum_{n=1}^{\infty} \frac{(2+\alpha)_{n}(a)_{n}}{(1+\alpha)_{n}(c)_{n}}\left|a_{1, n+1}\right|+(1-\lambda) \sum_{n=1}^{\infty} \frac{(2+\alpha)_{n}(a)_{n}}{(1+\alpha)_{n}(c)_{n}}\left|a_{2, n+1}\right| \\
& \quad \leqq 1 .
\end{aligned}
$$

This evidently completes the proof of Lemma 3.1. 
Since the class $\mathscr{V}_{0}(a, c ; \alpha)$ is convex, as we have shown in Lemma 3.1, it does have some extreme points given by Lemma 3.2 and Theorem 3.1 below.

\section{Lemma 3.2. Let}

$$
f_{0}(z)=z
$$

and

$$
f_{n}(z)=z-\frac{(1+\alpha)_{n}(c)_{n}}{(2+\alpha)_{n}(a)_{n}} z^{n+1} \quad(n \in \mathscr{N})
$$

with $(a)_{n} /(c)_{n}>0$. Then $f(z) \in \mathscr{V}_{0}(a, c ; \alpha)$ if and only if it can be expressed in the form

$$
f(z)=\sum_{n=0}^{\infty} \lambda_{n} f_{n}(z)
$$

where $\lambda_{n} \geqq 0, \forall n \in \mathscr{N} \cup\{0\}$, and

$$
\sum_{n=0}^{\infty} \lambda_{n}=1 .
$$

Remark 3.1. Stated simply, Lemma 3.2 asserts that the family $\mathscr{V}_{0}(a, c ; \alpha)$ is an infinite-dimensional simplex with definite vertices defined by certain extremal conditions.

Proof. We first assume that the function $f(z)$ has the expression (3.6), that is, that

$$
f(z)=z-\sum_{n=1}^{\infty} \frac{(1+\alpha)_{n}(c)_{n}}{(2+\alpha)_{n}(a)_{n}} \lambda_{n} z^{n+1} .
$$

Substituting from (3.8) into the left-hand side of (2.14), we have

$$
\sum_{n=1}^{\infty} \frac{(2+\alpha)_{n}(a)_{n}}{(1+\alpha)_{n}(c)_{n}} \frac{(1+\alpha)_{n}(c)_{n}}{(2+\alpha)_{n}(a)_{n}} \lambda_{n}=\sum_{n=1}^{\infty} \lambda_{n}=1-\lambda_{0} \leqq 1,
$$

which (in view of Theorem 2.3) implies that $f(z) \in \mathscr{V}_{0}(a, c ; \alpha)$.

For the converse, we assume that $f(z) \in \mathscr{V}_{0}(a, c ; \alpha)$ with $(a)_{n} /(c)_{n}>0$. Making use of Corollary 2.3, we may write

$$
\lambda_{n}=\frac{(2+\alpha)_{n}(a)_{n}}{(1+\alpha)_{n}(c)_{n}}\left|a_{n+1}\right| \quad(n \in \mathscr{N})
$$

and 


$$
\lambda_{0}=1-\sum_{n=1}^{\infty} \lambda_{n}
$$

We thus have the expression (3.6).

The following result on the extreme points of the class $\mathscr{V}_{0}(a, c ; \alpha)$ follows immediately from Lemma 3.2.

TheOREm 3.1. The extreme points of the class $\mathscr{V}_{0}(a, c ; \alpha)$ with $(a)_{n} /(c)_{n}$ $>0$ are the functions $f_{n}(z)(n \geqq 0)$ given by (3.4) and (3.5).

In precisely the same manner, we can establish

TheOREm 3.2. The extreme points of the class $\mathscr{W}_{0}(a, c ; \alpha)$ with $(a)_{n} /(c)_{n}$ $>0$ are the functions $f_{n}(z)(n \geqq 0)$ given by (3.4) and

$$
f_{n}(z)=z-\frac{(1)_{n}(1+\alpha)_{n}(c)_{n}}{(2)_{n}(2+\alpha)_{n}(a)_{n}} z^{n+1} \quad(n \in \mathscr{N}) .
$$

Remark 3.2. As already observed in Remark 3.1, each of Theorem 3.1 and Theorem 3.2 can indeed be restated in terms of infinite-dimensional simplexes.

Corresponding to Theorem 3.1 and Theorem 3.2, we now prove the following distortion theorems for functions belonging to the classes $\mathscr{V}_{0}(a, c ; \alpha)$ and $\mathscr{W}_{0}(a, c ; \alpha)$.

THEOREM 3.3. Let the function $f(z)$ defined by (1.26) be in the class $\mathscr{V}_{0}(a, c ; \alpha)$ with $(a)_{n} /(c)_{n}>0$ and $a \geqq c$. Then

$$
|z|-\frac{(1+\alpha) c}{(2+\alpha) a}|z|^{2} \leqq|f(z)| \leqq|z|+\frac{(1+\alpha) c}{(2+\alpha) a}|z|^{2}
$$

for $z \in \mathscr{U}$. Furthermore, if either

$$
\alpha \geqq 0 \quad \text { and } \quad c \leqq \min \left(\frac{1}{2} a, a-1\right)
$$

or

$$
-1<\alpha<0 \text { and } c>-1 \text {, }
$$

then

$$
1-\frac{2(1+\alpha) c}{(2+\alpha) a}|z| \leqq\left|f^{\prime}(z)\right| \leqq 1+\frac{2(1+\alpha) c}{(2+\alpha) a}|z|
$$

for $z \in \mathscr{U}$. The results (3.13) and (3.14) are sharp.

Proof. We first note that the extremal function is one of the extreme points. Since 


$$
\frac{(1+\alpha)_{n}(c)_{n}}{(2+\alpha)_{n}(a)_{n}}|z|^{n+1}
$$

is a decreasing function of $n$, we have, for $f_{n}(z)(n \geqq 0)$ defined by (3.4) and (3.5),

$$
f_{1}(|z|) \leqq\left|f_{n}(z)\right| \leqq-f_{1}(-|z|) \quad(z \in \mathscr{U})
$$

which readily yields the assertion (3.13) of Theorem 3.3.

To prove the assertion (3.14) of Theorem 3.3, we note that

$$
\left|f^{\prime}(z)\right| \geqq 1-\max _{n \in \mathcal{H}}\left\{\frac{(2)_{n}(1+\alpha)_{n}(c)_{n}}{(1)_{n}(2+\alpha)_{n}(a)_{n}}|z|^{n}\right\}
$$

and

$$
\left|f^{\prime}(z)\right| \leqq 1+\max _{n \in \mathscr{r}}\left\{\frac{(2)_{n}(1+\alpha)_{n}(c)_{n}}{(1)_{n}(2+\alpha)_{n}(a)_{n}}|z|^{n}\right\}
$$

for $z \in \mathscr{U}$. Thus we need only prove that the function

$$
G(a, c, \alpha, n,|z|)=\frac{(2)_{n}(1+\alpha)_{n}(c)_{n}}{(1)_{n}(2+\alpha)_{n}(a)_{n}}|z|^{n}
$$

is decreasing in $n(n \in \mathscr{N})$. For $|z| \neq 0$,

$$
G(a, c, \alpha, n,|z|) \geqq G(a, c, \alpha, n+1,|z|)
$$

if and only if

$$
\begin{aligned}
& G_{1}(a, c, \alpha, n,|z|) \\
& \quad=(n+1)(n+2+\alpha)(n+a)-(n+2)(n+1+\alpha)(n+c)|z| \\
& \quad \geqq 0 .
\end{aligned}
$$

Since $G_{1}(a, c, \alpha, n,|z|)$ is a decreasing function of $|z|$ for fixed $a, c, \alpha, n$, we have

$$
\begin{aligned}
& G_{1}(a, c, \alpha, n,|z|) \geqq G_{1}(a, c, \alpha, n, 1) \\
& \quad=(n+1)(a-c)(n+2+\alpha)-\alpha(n+c) \\
& \quad \geqq 0,
\end{aligned}
$$

provided that $a, c, \alpha$ are constrained to satisfy either set of the inequalities preceding (3.14). Appropriate substitutions in (3.16) and (3.17) lead to the assertion (3.14) of Theorem 3.3.

Finally, the bounds of (3.13) and (3.14) are attained by the function 


$$
f(z)=z-\frac{(1+\alpha) c}{(2+\alpha) a} z^{2}
$$

CoRollary 3.1. Let the function $f(z)$ defined by (1.26) be in the class $\mathscr{V}_{0}(a, c ; \alpha)$ with $(a)_{n} /(c)_{n}>0$ and $a \geqq c$. Then the unit disk $\mathscr{U}$ is mapped onto a domain that contains the disk $|w|<r_{0}$, where $r_{0}$ is given by

$$
r_{0}=1-\frac{(1+\alpha) c}{(2+\alpha) a}
$$

Corollary 3.2. Let the function $f(z)$ defined by (1.26) be in the class $\mathscr{V}_{0}(\alpha)$. Then

$$
|z|-\left(\frac{1+\alpha}{2+\alpha}\right)|z|^{2} \leqq|f(z)| \leqq|z|+\left(\frac{1+\alpha}{2+\alpha}\right)|z|^{2}
$$

for $z \in \mathscr{U}$. Furthermore

$$
1-\frac{2(1+\alpha)}{2+\alpha}|z| \leqq\left|f^{\prime}(z)\right| \leqq 1+\frac{2(1+\alpha)}{2+\alpha}|z|
$$

for $z \in \mathscr{U}$. The results (3.24) and (3.25) are sharp for the function

$$
f(z)=z-\left(\frac{1+\alpha}{2+\alpha}\right) z^{2}
$$

Remark 3.3. In its special case when $-1<\alpha \leqq 0$, Corollary 3.2 yields the corresponding distortion theorem for the class $\mathscr{T}^{*}(\alpha)$ proved by Silverman [14, p. 111, Theorem 4].

Applying the above technique mutatis mutandis, we can prove

THEOREM 3.4. Let the function $f(z)$ defined by (1.26) be in the class $\mathscr{W}_{0}(a, c ; \alpha)$ with $(a)_{n} /(c)_{n}>0$ and $a \geqq c$. Then

$$
|z|-\frac{(1+\alpha) c}{2(2+\alpha) a}|z|^{2} \leqq|f(z)| \leqq|z|+\frac{(1+\alpha) c}{2(2+\alpha) a}|z|^{2}
$$

for $z \in \mathscr{U}$. Furthermore

$$
1-\frac{(1+\alpha) c}{(2+\alpha) a}|z| \leqq\left|f^{\prime}(z)\right| \leqq 1+\frac{(1+\alpha) c}{(2+\alpha) a}|z|
$$

for $z \in \mathscr{U}$. The results (3.27) and (3.28) are sharp for the function

$$
f(z)=z-\frac{(1+\alpha) c}{2(2+\alpha) a} z^{2}
$$


Corollary 3.3. Let the function $f(z)$ defined by (1.26) be in the class $\mathscr{W}_{0}(a, c ; \alpha)$ with $(a)_{n} /(c)_{n}>0$ and $a \geqq c$. Then the unit disk $\mathscr{U}$ is mapped onto a domain that contains the disk $|w|<r_{1}$, where $r_{1}$ is given by

$$
r_{1}=1-\frac{(1+\alpha) c}{2(2+\alpha) a}
$$

Corollary 3.4. Let the function $f(z)$ defined by (1.26) be in the class $\mathscr{W}_{0}(\alpha)$. Then

$$
|z|-\frac{1+\alpha}{2(2+\alpha)}|z|^{2} \leqq|f(z)| \leqq|z|+\frac{1+\alpha}{2(2+\alpha)}|z|^{2}
$$

for $z \in \mathscr{U}$. Furthermore

$$
1-\left(\frac{1+\alpha}{2+\alpha}\right)|z| \leqq\left|f^{\prime}(z)\right| \leqq 1+\left(\frac{1+\alpha}{2+\alpha}\right)|z|
$$

for $z \in \mathscr{U}$. The results (3.31) and (3.32) are sharp for the function

$$
f(z)=z-\frac{1+\alpha}{2(2+\alpha)} z^{2} .
$$

\section{§4. Starlikeness and convexity}

For $-1<\alpha \leqq 0$, the classes $\mathscr{V}(\alpha)$ and $\mathscr{W}(\alpha)$ become, respectively, the class of starlike functions of order $\alpha$ and the class of convex functions of order $\alpha$. Naturally, therefore, we are interested in the starlikeness for functions belonging to the class $\mathscr{V}_{0}(a, c ; \alpha)$ and the convexity for functions belonging to the class $\mathscr{W}_{0}(a, c ; \alpha)$. We begin by proving

THEOREM 4.1. Let the function $f(z)$ defined by (1.26) be in the class $\mathscr{V}_{0}(a, c ; \alpha)$ with $(a)_{n} /(c)_{n}>0$. Then $f(z)$ is starlike of order $\delta(0 \leqq \delta<1)$ in the disk $|z|<r_{2}$, where

$$
r_{2}=\inf _{n \in \mathscr{r}}\left[\frac{(1-\delta)(2+\alpha)_{n}(a)_{n}}{(n+1-\delta)(1+\alpha)_{n}(c)_{n}}\right]^{1 / n} .
$$

Proof. It is sufficient to prove that

$$
\left|\frac{z f^{\prime}(z)}{f(z)}-1\right|<1-\delta
$$

for $|z|<r_{2}$. Note that 


$$
\left|\frac{z f^{\prime}(z)}{f(z)}-1\right| \leqq \frac{\sum_{n=1}^{\infty} n\left|a_{n+1}\right||z|^{n}}{1-\sum_{n=1}^{\infty}\left|a_{n+1}\right||z|^{n}} \leqq 1-\delta
$$

if and only if

$$
\sum_{n=1}^{\infty}(n+1-\delta)\left|a_{n+1}\right||z|^{n} \leqq 1-\delta .
$$

By means of Theorem 2.3, we need only find the values of $|z|$ for which

$$
|z|^{n} \leqq \frac{(1-\delta)(2+\alpha)_{n}(a)_{n}}{(n+1-\delta)(1+\alpha)_{n}(c)_{n}} \quad(n \in \mathscr{N}),
$$

which obviously completes the proof of Theorem 4.1.

Corollary 4.1. Let the function $f(z)$ defined by (1.26) be in the class $\mathscr{V}_{0}(\alpha)$. Then $f(z)$ is starlike of order $\delta(0 \leqq \delta<1)$ in the disk $|z|<r_{3}$, where

$$
r_{3}=\inf _{n \in \mathscr{r}}\left[\frac{(1-\delta)(2+\alpha)_{n}}{(n+1-\delta)(1+\alpha)_{n}}\right]^{1 / n} .
$$

Similarly, by applying Theorem 2.4 instead of Theorem 2.3, we have

TheOREM 4.2. Let the function $f(z)$ defined by (1.26) be in the class $\mathscr{W}_{0}(a, c ; \alpha)$ with $(a)_{n} /(c)_{n}>0$. Then $f(z)$ is convex of order $\delta(0 \leqq \delta<1)$ in the disk $|z|<r_{2}$, where $r_{2}$ is given by (4.1).

Corollary 4.2. Let the function $f(z)$ defined by (1.26) be in the class $\mathscr{W}_{0}(\alpha)$. Then $f(z)$ is convex of order $\delta(0 \leqq \delta<1)$ in the disk $|z|<r_{3}$, where $r_{3}$ is given by (4.6).

\section{§5. Further applications of Theorem 2.3 and Theorem 2.4}

We deduce several interesting relationships between the various subclasses of $\mathscr{A}$ as further consequences of Theorem 2.3 and Theorem 2.4. We first state

Theorem 5.1. Let $(a)_{n} /(c)_{n}>0$. Then

$$
\mathscr{W}_{0}(a, c ; \alpha) \subset \mathscr{V}_{0}\left(a, c ;-\frac{2}{3+\alpha}\right) .
$$

The result is the best possible. 
Proof. Let the function $f(z)$ defined by (1.26) belong to the class $\mathscr{W}_{0}(a, c ; \alpha)$ with $(a)_{n} /(c)_{n}>0$. Noting also that

$$
\frac{(2)_{n}(2+\alpha)_{n}}{(1)_{n}(1+\alpha)_{n}} \geqq \frac{\left(2-\frac{2}{3+\alpha}\right)_{n}}{\left(1-\frac{2}{3+\alpha}\right)_{n}}
$$

for $\alpha>-1$ and $n \in \mathscr{N}$, we thus find that

$$
\begin{aligned}
& \sum_{n=1}^{\infty} \frac{\left(2-\frac{2}{3+\alpha}\right)_{n}(a)_{n}}{\left(1-\frac{2}{3+\alpha}\right)_{n}(c)_{n}}\left|a_{n+1}\right| \\
& \quad \leqq \sum_{n=1}^{\infty} \frac{(2)_{n}(2+\alpha)_{n}(a)_{n}}{(1)_{n}(1+\alpha)_{n}(c)_{n}}\left|a_{n+1}\right| \leqq 1
\end{aligned}
$$

by Theorem 2.4. This implies that

$$
f(z) \in \mathscr{V}_{0}\left(a, c ;-\frac{2}{3+\alpha}\right),
$$

and, since the result is the best possible for the function $f(z)$ given by (3.29), we have completed the proof of Theorem 5.1.

\section{Corollary 5.1.}

$$
\mathscr{W}_{0}(\alpha) \subset \mathscr{V}_{0}\left(-\frac{2}{3+\alpha}\right)
$$

The result is the best possible for the function $f(z)$ given by (3.33).

THEOREM 5.2. Let $(a)_{n} /(c)_{n}>0 . \quad$ Then, if $a \geqq c$,

$$
\mathscr{V}_{0}(a, c ; \alpha) \subset \mathscr{V}_{0}(\alpha),
$$

and, if $a<c$,

$$
\mathscr{V}_{0}(a, c ; \alpha) \supset \mathscr{V}_{0}(\alpha) .
$$

Proof. Note that $(a)_{n} /(c)_{n}$ is an increasing function of $n$ if $a \geqq c$, and is decreasing in $n$ if $a<c$. By using Theorem 2.4 and Corollary 2.6, we complete the proof of Theorem 5.2.

Similarly, we have

Theorem 5.3. Let $(a)_{n} /(c)_{n}>0$. Then, if $a \geqq c$, 


$$
\mathscr{W}_{0}(a, c ; \alpha) \subset \mathscr{W}_{0}(\alpha)
$$

and, if $a<c$,

$$
\mathscr{W}_{0}(a, c ; \alpha) \supset \mathscr{W}_{0}(\alpha)
$$

§6. Subclasses of $\mathscr{V}_{0}(a, c ; \alpha)$ and $\mathscr{W}_{0}(a, c ; \alpha)$ with fixed finitely many coefficients

We introduce the subclasses $\mathscr{V}_{k}(a, c ; \alpha)$ and $\mathscr{W}_{k}(a, c ; \alpha)$ of analytic functions with fixed finitely many coefficients. In view of Corollary 2.3, we denote by $\mathscr{V}_{k}(a, c ; \alpha)$ the subclass of $\mathscr{V}_{0}(a, c ; \alpha)$ consisting of functions of the form

$$
f(z)=z-\sum_{i=1}^{k} A_{i} p_{i} z^{i+1}-\sum_{n=k+1}^{\infty} a_{n+1} z^{n+1} \quad\left(a_{n+1} \geqq 0\right),
$$

where

$$
\begin{aligned}
A_{i}= & \frac{(1+\alpha)_{i}(c)_{i}}{(2+\alpha)_{i}(a)_{i}}, \quad 0 \leqq p_{i} \leqq 1 \quad(i=1, \cdots, k), \\
& (a)_{n} /(c)_{n}>0, \quad \text { and } \quad 0 \leqq \sum_{i=1}^{k} p_{i} \leqq 1 .
\end{aligned}
$$

In view of Corollary 2.5, we also denote by $\mathscr{W}_{k}(a, c ; \alpha)$ the subclass of $\mathscr{W}_{0}(a, c ; \alpha)$ consisting of functions of the form

$$
f(z)=z-\sum_{i=1}^{k} B_{i} p_{i} z^{i+1}-\sum_{n=k+1}^{\infty} a_{n+1} z^{n+1} \quad\left(a_{n+1} \geqq 0\right),
$$

where

$$
\begin{gathered}
B_{i}=\frac{(1)_{i}(1+\alpha)_{i}(c)_{i}}{(2)_{i}(2+\alpha)_{i}(a)_{i}}, \quad 0 \leqq p_{i} \leqq 1 \quad(i=1, \cdots, k), \\
(a)_{n} /(c)_{n}>0, \quad \text { and } \quad 0 \leqq \sum_{i=1}^{k} p_{i} \leqq 1 .
\end{gathered}
$$

Moreover, we define the classes $\mathscr{V}_{k}(\alpha)$ and $\mathscr{W}_{k}(\alpha)$ by

$$
\mathscr{V}_{k}(\alpha)=\mathscr{V}_{k}(a, a ; \alpha)
$$

and

$$
\mathscr{W}_{k}(\alpha)=\mathscr{W}_{k}(a, a ; \alpha) .
$$

THEOREM 6.1. Let the function $f(z)$ be defined by (6.1). Then $f(z) \in$ $\mathscr{V}_{k}(a, c ; \alpha)$ if and only if 


$$
\sum_{n=k+1}^{\infty} \frac{(2+\alpha)_{n}(a)_{n}}{(1+\alpha)_{n}(c)_{n}} a_{n+1} \geqq 1-\sum_{i=1}^{k} p_{i} .
$$

The result (6.7) is sharp.

Proof. Putting

$$
a_{i}=A_{i} p_{i} \quad(i=1, \cdots, k)
$$

in (2.14), we have (6.7). Further, the result (6.7) is sharp for the function of the form

$$
\begin{aligned}
f(z)= & z-\sum_{i=1}^{k} A_{i} p_{i} z^{i+1} \\
& -\frac{(1+\alpha)_{n}(c)_{n}\left(1-\sum_{i=1}^{k} p_{i}\right)}{(2+\alpha)_{n}(a)_{n}} z^{n+1} \quad(n \geqq k+1) .
\end{aligned}
$$

Corollary 6.1. Let the function $f(z)$ be defined by (6.1) with $a=c$. Then $f(z) \in \mathscr{V}_{k}(\alpha)$ if and only if

$$
\sum_{n=k+1}^{\infty} \frac{(2+\alpha)_{n}}{(1+\alpha)_{n}} a_{n+1} \leqq 1-\sum_{i=1}^{k} p_{i} .
$$

The result (6.10) is sharp for the function

$$
\begin{aligned}
f(z)= & z-\sum_{i=1}^{k} \frac{(1+\alpha)_{i} p_{i}}{(2+\alpha)_{i}} z^{i+1} \\
& -\frac{(1+\alpha)_{n}\left(1-\sum_{i=1}^{k} p_{i}\right)}{(2+\alpha)_{n}} z^{n+1} \quad(n \geqq k+1) .
\end{aligned}
$$

THEOREM 6.2. Let the function $f(z)$ be defined by (6.3). Then $f(z) \in$ $\mathscr{W}_{k}(a, c ; \alpha)$ if and only if

$$
\sum_{n=k+1}^{\infty} \frac{(2)_{n}(2+\alpha)_{n}(a)_{n}}{(1)_{n}(1+\alpha)_{n}(c)_{n}} a_{n+1} \leqq 1-\sum_{i=1}^{k} p_{i} .
$$

The result (6.12) is sharp for the function

$$
\begin{aligned}
f(z) & =z-\sum_{i=1}^{k} B_{i} p_{i} z^{i+1} \\
- & -\frac{(1)_{n}(1+\alpha)_{n}(c)_{n}\left(1-\sum_{i=1}^{k} p_{i}\right)}{(2)_{n}(2+\alpha)_{n}(a)_{n}} z^{n+1} \quad(n \geqq k+1) .
\end{aligned}
$$

The proof of Theorem 6.2 is much akin to that of Theorem 6.1, and we omit the details involved. 
Corollary 6.2. Let the function $f(z)$ be defined by (6.3) with $a=c$. Then $f(z) \in \mathscr{W}_{k}(\alpha)$ if and only if

$$
\sum_{n=k+1}^{\infty} \frac{(2)_{n}(2+\alpha)_{n}}{(1)_{n}(1+\alpha)_{n}} a_{n+1} \leqq 1-\sum_{i=1}^{k} p_{i} .
$$

The result (6.14) is sharp for the function

$$
\begin{aligned}
f(z)= & z-\sum_{i=1}^{k} \frac{(1)_{i}(1+\alpha)_{i} p_{i}}{(2)_{i}(2+\alpha)_{i}} z^{i+1} \\
& -\frac{(1)_{n}(1+\alpha)_{n}\left(1-\sum_{i=1}^{k} p_{i}\right)}{(2)_{n}(2+\alpha)_{n}} z^{n+1} \quad(n \geqq k+1) .
\end{aligned}
$$

With the aid of Theorem 6.1 and Theorem 6.2, we can find the extreme points of the classes $\mathscr{V}_{k}(a, c ; \alpha)$ and $\mathscr{W}_{k}(a, c ; \alpha)$ by using the techniques detailed in Section 3.

THEOREM 6.3. The extreme points of the class $\mathscr{V}_{k}(a, c ; \alpha)$ with $(a)_{n} /(c)_{n}$ $>0$ are

$$
f_{k+1}(z)=z-\sum_{i=1}^{k} A_{i} p_{i} z^{i+1}
$$

and

$$
\begin{aligned}
f_{n+1}(z)= & z-\sum_{i=1}^{k} A_{i} p_{i} z^{i+1} \\
& -\frac{(1+\alpha)_{n}(c)_{n}\left(1-\sum_{i=1}^{k} p_{i}\right)}{(2+\alpha)_{n}(a)_{n}} z^{n+1} \quad(n \geqq k+1),
\end{aligned}
$$

where $A_{i}$ is given by (6.2).

TheOREM 6.4. The extreme points of the class $\mathscr{W}_{k}(a, c ; \alpha)$ with $(a)_{n} /(c)_{n}$ $>0$ are

$$
f_{k+1}(z)=z-\sum_{i=1}^{k} B_{i} p_{i} z^{i+1}
$$

and

$$
\begin{aligned}
f_{n+1}(z) & =z-\sum_{i=1}^{k} B_{i} p_{i} z^{i+1} \\
& -\frac{(1)_{n}(1+\alpha)_{n}(c)_{n}\left(1-\sum_{i=1}^{k} p_{i}\right)}{(2)_{n}(2+\alpha)_{n}(a)_{n}} z^{n+1} \quad(n \geqq k+1),
\end{aligned}
$$

where $B_{i}$ is given by (6.4). 


\section{§7. Characterization theorems involving the functional $\mathscr{J}_{\beta}(f)$}

Let $\mathscr{J}_{\beta}(f)$ denote a functional defined by

$$
\mathscr{J}_{\beta}(f)=\frac{\beta+1}{z^{\beta}} \int_{0}^{z} t^{\beta-1} f(t) d t
$$

for $f(z) \in \mathscr{A}$ and for a real number $\beta>-1$. The functional $\mathscr{J}_{\beta}(f)$, when $\beta \in \mathscr{N}$, was studied by Bernardi [1]. In particular, the functional $\mathscr{J}_{1}(f)$ was considered earlier by Libera [5] and Livingston [6].

Remark 7.1. Clearly, $\mathscr{J}_{\beta}(f)$ defined by (7.1) is a particular solution of the ordinary first-order linear differential equation

$$
\operatorname{tg}^{\prime}(t)+\beta g(t)=(\beta+1) f(t)
$$

at the point $t=z$. Furthermore, by comparing (7.1) and (1.12), we have

$$
\mathscr{J}_{\beta}(f)=\mathscr{L}(\beta+2, \beta+1) f .
$$

Thus, although some of the statements of this section can alternatively be proved by using this simple relationship together with the obvious fact that

$$
\frac{\beta+1}{\beta+2}>0 \quad \text { for } \beta>-1
$$

our demonstration of the characterization theorems involving the functional $\mathscr{J}_{\beta}(f)$ will be based upon the following result.

LEMMA 7.1 (Jack [4]). Let $w(z)$ be regular in the unit disk $\mathscr{U}$ with $w(0)=0$. Then, if $|w(z)|$ attains its maximum value on the circle $|z|=r$ $(0 \leqq r<1)$ at a point $z_{0}$, we can write

$$
\boldsymbol{z}_{0} w^{\prime}\left(\boldsymbol{z}_{0}\right)=m w\left(\boldsymbol{z}_{0}\right),
$$

where $m$ is real and $m \geqq 1$.

We now state our first characterization theorem involving the functional $\mathscr{J}_{\beta}(f)$.

THEOREM 7.1. Let the function $f(z)$ defined by (1.1) by in the class $\mathscr{V}(a, c ; \alpha)$. Then, for $\beta \geqq \alpha>-1, \mathscr{J}_{\beta}(f)$ is also in the class $\mathscr{V}(a, c ; \alpha)$.

Proof. Define the function $w(z)$ by

$$
\frac{z\left[\mathscr{L}(a, c) \mathscr{J}_{\beta}(f)\right]^{\prime}}{\mathscr{L}(a, c) \mathscr{J}_{\beta}(f)}=\frac{1-(1+2 \alpha) w(z)}{1+w(z)} \quad[w(z) \neq-1] .
$$

Then it is clear that $w(0)=0$. 
Differentiating both sides of (7.3) logarithmically, we have

$$
\begin{gathered}
\frac{z^{2}\left[\mathscr{L}(a, c) \mathscr{J}_{\beta}(f)\right]^{\prime \prime}+z\left[\mathscr{L}(a, c) \mathscr{J}_{\beta}(f)\right]^{\prime}}{z\left[\mathscr{L}(a, c) \mathscr{J}_{\beta}(f)\right]^{\prime}}-\frac{z\left[\mathscr{L}(a, c) \mathscr{J}_{\beta}(f)\right]^{\prime}}{\mathscr{L}(a, c) \mathscr{J}_{\beta}(f)} \\
\quad=-\frac{2(1+\alpha) z w^{\prime}(z)}{[1+w(z)][1-(1+2 \alpha) w(z)]}
\end{gathered}
$$

Note that

$$
z\left[\mathscr{L}(a, c) \mathscr{J}_{\beta}(f)\right]^{\prime}=(\beta+1) \mathscr{L}(a, c) f(z)-\beta \mathscr{L}(a, c) \mathscr{J}_{\beta}(f),
$$

which readily yields

$$
z^{2}\left[\mathscr{L}(a, c) \mathscr{J}_{\beta}(f)\right]^{\prime \prime}=(\beta+1) z[\mathscr{L}(a, c) f(z)]^{\prime}-(\beta+1) z\left[\mathscr{L}(a, c) \mathscr{J}_{\beta}(f)\right]^{\prime} .
$$

Substituting from (7.5) and (7.6) into (7.4), we have

$$
\begin{gathered}
\frac{(\beta+1) z[\mathscr{L}(a, c) f(z)]^{\prime}}{z\left[\mathscr{L}(a, c) \mathscr{J}_{\beta}(f)\right]^{\prime}}-\frac{(\beta+1) \mathscr{L}(a, c) f(z)}{\mathscr{L}(a, c) \mathscr{J}_{\beta}(f)} \\
\quad=-\frac{2(1+\alpha) z w^{\prime}(z)}{[1+w(z)][1-(1+2 \alpha) w(z)]}
\end{gathered}
$$

or, equivalently,

$$
\begin{aligned}
& \frac{(\beta+1) \mathscr{L}(a, c) f(z)}{z\left[\mathscr{L}(a, c) \mathscr{J}_{\beta}(f)\right]^{\prime}}\left[\frac{z[\mathscr{L}(a, c) f(z)]^{\prime}}{\mathscr{L}(a, c) f(z)}-\frac{z\left[\mathscr{L}(a, c) \mathscr{J}_{\beta}(f)\right]^{\prime}}{\mathscr{L}(a, c) \mathscr{J}_{\beta}(f)}\right] \\
& \quad=-\frac{2(1+\alpha) z w^{\prime}(z)}{[1+w(z)][1-(1+2 \alpha) w(z)]}
\end{aligned}
$$

It follows from (7.5) that

$$
\begin{aligned}
\frac{(\beta+1) \mathscr{L}(a, c) f(z)}{z\left[\mathscr{L}(a, c) \mathscr{J}_{\beta}(f)\right]^{\prime}} & =1+\frac{\beta \mathscr{L}(a, c) \mathscr{J}_{\beta}(f)}{z\left[\mathscr{L}(a, c) \mathscr{J}_{\beta}(f)\right]^{\prime}} \\
& =\frac{(\beta+1)-(1+2 \alpha-\beta) w(z)}{1-(1+2 \alpha) w(z)},
\end{aligned}
$$

which, in conjunction with (7.8), yields

$$
\begin{aligned}
\frac{z[\mathscr{L}(a, c) f(z)]^{\prime}}{\mathscr{L}(a, c) f(z)}= & \frac{1-(1+2 \alpha) w(z)}{1+w(z)} \\
& -\frac{2(1+\alpha) z w^{\prime}(z)}{[1+w(z)][(\beta+1)-(1+2 \alpha-\beta) w(z)]}
\end{aligned}
$$

Assume that there exists a point $z_{0} \in \mathscr{U}$ such that

$$
\max _{|z| \leqq\left|z_{0}\right|}|w(z)|=\left|w\left(z_{0}\right)\right|=1 \quad\left[w\left(z_{0}\right) \neq-1\right] .
$$


Then, by using Lemma 7.1 , we can write

$$
z_{0} w^{\prime}\left(z_{0}\right)=m w\left(z_{0}\right) \quad(m \text { is real and } m \geqq 1) .
$$

Applying Lemma 7.1 to $w(z)$ at $z_{0}$, and putting $w\left(z_{0}\right)=e^{i \theta_{0}}(i=\sqrt{-1})$, we observe that

$$
\begin{aligned}
\operatorname{Re}\left\{\frac{z\left[\mathscr{L}(a, c) f\left(\boldsymbol{z}_{0}\right)\right]^{\prime}}{\mathscr{L}(a, c) f\left(\boldsymbol{z}_{0}\right)}\right\}= & \operatorname{Re}\left\{\frac{1-(1+2 \alpha) w\left(\boldsymbol{z}_{0}\right)}{1+w\left(z_{0}\right)}\right\} \\
& -\operatorname{Re}\left\{\frac{2(1+\alpha) m w\left(z_{0}\right)}{\left[1+w\left(z_{0}\right)\right]\left[(\beta+1)-(1+2 \alpha-\beta) w\left(z_{0}\right)\right]}\right\} \\
= & -\alpha-\frac{m}{\Delta}(1+\alpha)(\beta-\alpha)\left(1+\cos \theta_{0}\right) \leqq-\alpha,
\end{aligned}
$$

where, for convenience,

$$
\begin{aligned}
\Delta= & {\left[(\beta-\alpha)\left(1+\cos \theta_{0}\right)+(1+2 \alpha-\beta) \sin ^{2} \theta_{0}\right]^{2} } \\
& +\sin ^{2} \theta_{0}\left[(\beta-\alpha)-(1+2 \alpha-\beta) \cos \theta_{0}\right]^{2} .
\end{aligned}
$$

Clearly, (7.11) contradicts the hypothesis that $f(z) \in \mathscr{V}(a, c ; \alpha)$. Hence $|w(z)|$ $<1$ for $z \in \mathscr{U}$, which implies that

$$
\operatorname{Re}\left\{\frac{z\left[\mathscr{L}(a, c) \mathscr{J}_{\beta}(f)\right]^{\prime}}{\mathscr{L}(a, c) \mathscr{J}_{\beta}(f)}\right\}=\operatorname{Re}\left\{\frac{1-(1+2 \alpha) w(z)}{1+w(z)}\right\}>-\alpha
$$

that is, that $\mathscr{J}_{\beta}(f) \in \mathscr{V}(a, c ; \alpha)$. This evidently completes the proof of Theorem 7.1.

Corollary 7.1. Let the function $f(z)$ defined by (1.1) be in the class $\mathscr{V}(a, c ; \alpha)$. Then, for $\beta \geqq \alpha>-1$,

$$
\mathscr{L}(a, c) \mathscr{J}_{\beta}(f) \in \mathscr{S}^{*} \quad \text { and } \quad \mathscr{J}_{\beta}(\mathscr{L}(a, c) f(z)) \in \mathscr{S}^{*} .
$$

Proof. The first assertion in (7.14) follows easily from Theorem 7.1. Noting that

$$
\mathscr{L}(a, c) \mathscr{J}_{\beta}(f)=\mathscr{J}_{\beta}(\mathscr{L}(a, c) f(z)),
$$

we have the second assertion in (7.14).

Theorem 7.2. Let the function $f(z)$ defined by (1.1) be in the class $\mathscr{W}(a, c ; \alpha)$. Then, for $\beta \geqq \alpha>-1, \mathscr{J}_{\beta}(f)$ is also in the class $\mathscr{W}(a, c ; \alpha)$.

Proof. Since

$$
f(z) \in \mathscr{W}(a, c ; \alpha) \Longleftrightarrow z f^{\prime}(z) \in \mathscr{V}(a, c ; \alpha),
$$


we need only prove that

$$
z\left[\mathscr{J}_{\beta}(f)\right]^{\prime} \in \mathscr{V}(a, c ; \alpha) .
$$

Indeed, with the aid of Theorem 7.1, we observe that

$$
\begin{aligned}
f(z) \in \mathscr{W}(a, c ; \alpha) & \Longleftrightarrow z f^{\prime}(z) \in \mathscr{V}(a, c ; \alpha) \\
& \Longrightarrow \mathscr{J}_{\beta}\left(z f^{\prime}(z)\right) \in \mathscr{V}(a, c ; \alpha) \\
& \Longrightarrow z\left[\mathscr{J}_{\beta}(f)\right]^{\prime} \in \mathscr{V}(a, c ; \alpha),
\end{aligned}
$$

which completes the proof of Theorem 7.2.

CoROllary 7.2. Let the function $f(z)$ defined by (1.1) be in the class $\mathscr{W}(a, c ; \alpha)$. Then, for $\beta \geqq \alpha>-1$,

$$
\mathscr{L}(a, c) \mathscr{J}_{\beta}(f) \in \mathscr{K} \quad \text { and } \quad \mathscr{J}_{\beta}(\mathscr{L}(a, c) f(z)) \in \mathscr{K} .
$$

\section{§8. Applications of the fractional calculus operator $\Omega^{\lambda}$}

From among the various definitions of fractional calculus (that is, fractional derivatives and fractional integrals) given in the literature cited, we choose to recall here the following definitions which were used recently by Owa [8] (and by Srivastava and Owa [17]):

Definition 8.1. The fractional integral of order $\lambda$ is defined, for a function $f(z)$, by

$$
D_{z}^{-\lambda} f(z)=\frac{1}{\Gamma(\lambda)} \int_{0}^{z} \frac{f(\zeta)}{(z-\zeta)^{1-\lambda}} d \zeta,
$$

where $\lambda>0, f(z)$ is an analytic function in a simply-connected region of the $z$-plane containing the origin, and the multiplicity of $(z-\zeta)^{\lambda-1}$ is removed by requiring $\log (z-\zeta)$ to be real when $z-\zeta>0$.

Definition 8.2. The fractional derivative of order $\lambda$ is defined, for a function $f(z)$, by

$$
D_{z}^{\lambda} f(z)=\frac{1}{\Gamma(1-\lambda)} \frac{d}{d z} \int_{0}^{z} \frac{f(\zeta)}{(z-\zeta)^{\lambda}} d \zeta,
$$

where $0 \leqq \lambda<1, f(z)$ is an analytic function in a simply-connected region of the $z$-plane containing the origin, and the multiplicity of $(z-\zeta)^{-\lambda}$ is removed as in Definition 8.1 above.

Definition 8.3. Under the hypotheses of Definition 8.2, the fractional derivative of order $n+\lambda$ is defined by 


$$
D_{z}^{n+\lambda} f(z)=\frac{d^{n}}{d z^{n}} D_{z}^{2} f(z)
$$

where $0 \leqq \lambda<1$, and $n \in \mathscr{N} \cup\{0\}$.

Following the lines of Owa and Srivastava [9], we introduce the linear operator $\Omega^{\lambda}$ defined by

$$
\Omega^{\lambda} f=\Gamma(2-\lambda) z^{\lambda} D_{z}^{\lambda} f(z) \quad(\lambda<1)
$$

for functions $f(z)$ belonging to the class $\mathscr{A}$. Note that

$$
\Omega^{\lambda} f=\mathscr{L}(2,2-\lambda) f \quad(\lambda<1)
$$

for functions $f(z)$ defined by (1.1). Further, we define

$$
\Omega f \equiv \Omega^{1} f=z f^{\prime}(z)=\mathscr{L}(2,1) f
$$

and

$$
\Omega^{n} f=\Omega\left(\Omega^{n-1} f\right)=\mathscr{L}^{n}(2,1) f \quad(n \in \mathscr{N}),
$$

with, of course, $\Omega^{\circ} f=f(z)$. Then it is easily observed that

$$
\Omega^{n+\lambda} f=\Omega^{n}\left(\Omega^{\lambda} f\right)=\mathscr{L}^{n}(2,1) \mathscr{L}(2,2-\lambda) f=\Omega^{\lambda}\left(\Omega^{n} f\right)
$$

for $\lambda<1$ and $n \in \mathscr{N}$.

We begin by proving

TheOREm 8.1. Let the function $f(z)$ defined by (1.26) be in the class $\mathscr{V}_{0}(2,2-\lambda ; \alpha)$ for $\lambda<1$. Then

$$
\left|D_{z}^{\lambda} f(z)\right| \geqq \frac{|z|^{1-\lambda}}{\Gamma(2-\lambda)}\left\{1-\left(\frac{1+\alpha}{2+\alpha}\right)|z|\right\}
$$

and

$$
\left|D_{z}^{2} f(z)\right| \leqq \frac{|z|^{1-\lambda}}{\Gamma(2-\lambda)^{4}}\left\{1+\left(\frac{1+\alpha}{2+\alpha}\right)|z|\right\}
$$

for $z \in \mathscr{U}$. The results (8.9) and (8.10) are sharp.

Proof. Since $f(z) \in \mathscr{V}_{0}(2,2-\lambda ; \alpha)$, the definition of $\mathscr{V}_{0}(2,2-\lambda ; \alpha)$ and (8.5) give

$$
\Omega^{\lambda} f=\mathscr{L}(2,2-\lambda) f \in \mathscr{V}_{0}(\alpha) .
$$

Now the assertions (8.9) and (8.10) of Theorem 8.1 follow immediately from (8.4) and Corollary 3.2. 
The results (8.9) and (8.10) are sharp for the function

$$
f(z)=z-\frac{(1+\alpha)(2-\lambda)}{2(2+\alpha)} z^{2} .
$$

Similarly, we have

THEOREM 8.2. Let the function $f(z)$ defined by (1.26) be in the class $\mathscr{W}_{0}(2,2-\lambda ; \alpha)$ for $\lambda<1$. Then

$$
\left|D_{z}^{\lambda} f(z)\right| \geqq \frac{|z|^{1-\lambda}}{\Gamma(2-\lambda)}\left(1-\frac{1+\alpha}{2(2+\alpha)}|z|\right)
$$

and

$$
\left|D_{z}^{2} f(z)\right| \leqq \frac{\mid z^{1-\lambda}}{\Gamma(2-\lambda)}\left(1+\frac{1+\alpha}{2(2+\alpha)}|z|\right)
$$

for $z \in \mathscr{U}$. The results (8.13) and (8.14) are sharp for the function

$$
f(z)=z-\frac{(1+\alpha)(2-\lambda)}{4(2+\alpha)} z^{2} .
$$

TheOREm 8.3. Let $\lambda<1$. Then

$$
\Omega^{1+\lambda} \mathscr{W}(2,2-\lambda ; \alpha) \subset \mathscr{V}(\alpha) .
$$

Proof. If $f(z) \in \mathscr{W}(2,2-\lambda ; \alpha)$, then

$$
\Omega^{\lambda} f=\mathscr{L}(2,2-\lambda) f \in \mathscr{W}(\alpha) .
$$

Hence we have

$$
\Omega^{2} \mathscr{W}(2,2-\lambda ; \alpha) \subset \mathscr{W}(\alpha)
$$

Applying the linear operator $\mathscr{L}(2,1)$ in $(8.17)$, and using $(1.4)$, we observe that

$$
\mathscr{L}(2,1) \Omega^{\lambda} \mathscr{W}(2,2-\lambda ; \alpha) \subset \mathscr{V}(\alpha),
$$

which implies (8.16).

CoROLlary 8.1. Let $\lambda<1$. Then

$$
\Omega^{1+\lambda \mathscr{W}_{0}}(2,2-\lambda ; \alpha) \subset \mathscr{V}_{0}(\alpha) .
$$

Corollary 8.2. Let $\lambda<1$. Then

$$
\Omega^{1+\lambda \mathscr{W}_{k}}(2,2-\lambda ; \alpha) \subset \mathscr{V}_{k}(\alpha) \text {. }
$$

Next we prove 
TheOREM 8.4. Let $\lambda<1$. Then

$$
\Omega^{\lambda} \mathscr{W}(1,2-\lambda ; \alpha) \subset \mathscr{V}(\alpha)
$$

Proof. Since $f(z) \in \mathscr{W}(1,2-\lambda ; \alpha)$ implies that $f(z) \in \mathscr{L}(2-\lambda, 1) \mathscr{W}(\alpha)$, we have

$$
\begin{aligned}
\Omega^{2} \mathscr{W}(1,2-\lambda ; \alpha) & \subset \Omega^{\lambda} \mathscr{L}(2-\lambda, 1) \mathscr{W}(\alpha) \\
& =\mathscr{L}(2,2-\lambda) \mathscr{L}(2-\lambda, 1) \mathscr{W}(\alpha) \\
& =\mathscr{L}(2,1) \mathscr{W}(\alpha)=\mathscr{V}(\alpha),
\end{aligned}
$$

by using the properties of the linear operator involved.

Corollary 8.3. Let $\lambda<1$. Then

$$
\Omega^{\lambda} \mathscr{W}_{0}(1,2-\lambda ; \alpha) \subset \mathscr{V}_{0}(\alpha) .
$$

Corollary 8.4. Let $\lambda<1$. Then

$$
\Omega^{\lambda} \mathscr{W}_{k}(1,2-\lambda ; \alpha) \subset \mathscr{V}_{k}(\alpha) .
$$

Finally, we prove the following theorem involving generalized hypergeometric functions.

TheOREM 8.5. Let $\lambda<1$. Then

$$
z_{q+2} F_{q+1}(2, \cdots, 2,2 ; 1, \cdots, 1,2-\lambda ; z) \in \Omega^{q+1+\lambda}\left(\mathscr{S} *\left(\frac{1}{2}\right) \cap \mathscr{K}\right) .
$$

Proof. Note that

$$
\begin{aligned}
& z_{q+2} F_{q+1}(2, \cdots, 2,2 ; 1, \cdots, 1,2-\lambda ; z) \\
& \quad=\mathscr{L}^{q+1}(2,1) \mathscr{L}(2,2-\lambda) \phi(1,1 ; z) \\
& \quad=\Omega^{q+1+\lambda} \phi(1,1 ; z)
\end{aligned}
$$

where $\phi(1,1 ; z)$ is defined by (1.10). It is easy to show that

$$
\phi(1,1 ; z) \in \mathscr{S}^{*}\left(\frac{1}{2}\right) \cap \mathscr{K},
$$

which obviously completes the proof of Theorem 8.5.

\section{REFERENCES}

[1] S. D. Bernardi, Convex and starlike univalent functions, Trans. Amer. Math. Soc., 135 (1969), 429-446.

[2] B. C. Carlson and D. B. Shaffer, Starlike and prestarlike hypergeometric functions, SIAM J. Math. Anal., 15 (1984), 737-745.

[ 3 ] A. Erdélyi, W. Magnus, F. Oberhettinger and F. G. Tricomi, Tables of Integral Transforms, Vol. II, McGraw-Hill, New York, London and Toronto, 1954. 
[4] I. S. Jack, Functions starlike and convex of order $\alpha$, J. London Math. Soc. (2), 3 (1971), 469-474.

[ 5 ] R. J. Libera, Some classes of regular univalent functions, Proc. Amer. Math. Soc., 16 (1965), 755-758.

[6] A. E. Livingston, On the radius of univalence of certain analytic functions, Proc. Amer. Math. Soc., 17 (1966), 352-357.

[ 7 ] T. H. MacGregor, The radius of convexity for starlike functions of order $\frac{1}{2}$, Proc. Amer. Math. Soc., 14 (1963), 71-76.

[ 8 ] S. Owa, On the distortion theorems. I, Kyungpook Math. J., 18 (1978), 53-59.

[ 9 ] S. Owa and H. M. Srivastava, Univalent and starlike generalized hypergeometric functions, Canad. J. Math. (to appear).

[10] B. Pinchuk, On starlike and convex functions of order $\alpha$, Duke Math. J., 35 (1968), $721-734$.

[11] M. S. Robertson, On the theory of univalent functions, Ann. of Math., 37 (1936), $374-408$.

[12] B. Ross, A brief history and exposition of the fundamental theory of fractional calculus, in Fractional Calculus and Its Applications (B. Ross, ed.), SpringerVerlag, Berlin, Heidelberg and New York, 1975, pp. 1-36.

[13] A. Schild, On starlike functions of order $\alpha$, Amer. J. Math., 87 (1965), 65-70.

[14] H. Silverman, Univalent functions with negative coefficients, Proc. Amer. Math. Soc., 51 (1975), 109-116.

[15] H. M. Srivastava and R. G. Buschman, Convolution Integral Equations with Special Function Kernels, John Wiley and Sons, New York, London, Sydney and Toronto, 1977.

[16] H. M. Srivastava and B. R. K. Kashyap, Special Functions in Queuing Theory and Related Stochastic Processes, Academic Press, New York and London, 1982.

[17] H. M. Srivastava and S. Owa, An application of the fractional derivative, Math. Japon., 29 (1984), 383-389.

[18] H. M. Srivastava, S. Owa and K. Nishimoto, Some fractional differintegral equations, J. Math. Anal. Appl., 106 (1985), 360-366.

H. M. Srivastava

Department of Mathematics

University of Victoria

Victoria, British Columbia V8W $2 Y 2$

Canada

Shigeyoshi Owa

Department of Mathematics

Kinki University

Higashi-Osaka, Osaka 577

Japan 